\title{
Fornix Integrity and Hippocampal Volume in Male Schizophrenic Patients
}

\section{Citation}

Kuroki, Noriomi, Marek Kubicki, Paul G. Nestor, Dean F. Salisbury, Hae-Jeong Park, James J. Levitt, Sophie Woolston, et al. 2006. "Fornix Integrity and Hippocampal Volume in Male Schizophrenic Patients." Biological Psychiatry 60 (1) (July): 22-31. doi:10.1016/ j.biopsych.2005.09.021.

\section{Published Version}

doi:10.1016/j.biopsych.2005.09.021

\section{Permanent link}

http://nrs.harvard.edu/urn-3:HUL.InstRepos:28566444

\section{Terms of Use}

This article was downloaded from Harvard University's DASH repository, and is made available under the terms and conditions applicable to Other Posted Material, as set forth at http:// nrs.harvard.edu/urn-3:HUL.InstRepos:dash.current.terms-of-use\#LAA

\section{Share Your Story}

The Harvard community has made this article openly available.

Please share how this access benefits you. Submit a story.

\section{Accessibility}




\title{
Fornix Integrity and Hippocampal Volume in Male Schizophrenic
}

\section{Patients}

\author{
Noriomi Kuroki, Marek Kubicki, Paul G. Nestor, Dean F. Salisbury, Hae-Jeong Park, James \\ J. Levitt, Sophie Woolston, Melissa Frumin, Margaret Niznikiewicz, Carl-Fredrik Westin, \\ Stephan E. Maier, Robert W. McCarley, and Martha E. Shenton \\ Clinical Neuroscience Division (NK, MK, PGN, DFS, H-JP, JJL, SW, MF, MN, MWM, MES), \\ Laboratory of Neuroscience, Department of Psychiatry, Boston VA Healthcare System, Brockton \\ Division, and Harvard Medical School, Brockton, Massachusetts; Department of Psychology (DGN), \\ University of Massachusetts, Boston; Cognitive Neuroscience Laboratory (DFS), McLean Hospital, \\ Belmont; and Surgical Planning Laboratory (C-FW, SEM, MES), MRI Division, Brigham and \\ Women's Hospital, and Department of Radiology, Harvard Medical School, Boston, Massachusetts.
}

\begin{abstract}
Background-The hippocampus has been shown to be abnormal in schizophrenia. The fornix is one of the main fiber tracts connecting the hippocampus with other brain regions. Few studies have evaluated the fornix in schizophrenia, however. A focus on fornix abnormalities and their association with hippocampal abnormalities might figure importantly in our understanding of the pathophysiology of schizophrenia.

Methods-Line-scan diffusion tensor imaging (DTI) was used to evaluate diffusion in the fornix in 24 male patients with chronic schizophrenia and 31 male control subjects. Maps of fractional anisotropy (FA) and mean diffusivity $\left(\mathrm{D}_{\mathrm{m}}\right)$, which are indices sensitive to white-matter integrity, were generated to quantify diffusion within the fornix. We used high spatial resolution magnetic resonance imaging (MRI) to measure hippocampal volume.
\end{abstract}

Results-FA and cross-sectional area of the fornix were significantly reduced in patients compared with control subjects. $\mathrm{D}_{\mathrm{m}}$ was significantly increased, whereas hippocampal volume was bilaterally reduced in patients. Reduced hippocampal volume was correlated with increased mean $D_{m}$ and reduced cross-sectional area of the fornix for patients. Patients also showed a significant correlation between reduced scores on neuropsychologic measures of declarative-episodic memory and reduced hippocampal volumes.

Conclusions-These findings demonstrate a disruption in fornix integrity in patients with schizophrenia.

\section{Keywords}

Anisotropy; diffusion tensor imaging; fornix; hippocampus; MRI; white matter

Medial temporal lobe regions have been well studied in schizophrenia (Shenton et al 2001). The hippocampus, thought to play a crucial role in the pathology of schizophrenia, has been reported as abnormal in several reports (Benes 1999; Schmajuk 2001; Weinberger 1999). The fornix, in addition to the entorhinal cortex, is a main pathway linking the hippocampus with 
other brain regions. Abnormal connections between the hippocampus and other brain regions might therefore figure prominently in understanding the pathophysiology of schizophrenia. Few studies have evaluated the fornix in schizophrenia, however.

Of particular note, pyramidal cell axons from the subiculum and Ammon's horn enter the fimbria via the alveus and then enter the fornix. Separately, the left and right fornix, exiting the left and right hippocampi, form the crux of the fornix, which ascends below the splenium of the corpus callosum, and then merge to form the body of the fornix. Most axons are ipsilateral, but some cross in the body of the fornix. The fornix then separates again into left and right columns above the anterior commissure and descends just posterior to the anterior commissure (e.g., postcommissural fornix) but also sends branches (e.g., precommissural fornix) to other regions (i.e., nucleus accumbens).

Axons arising from Ammon's horn go to the septal regions and the subiculum. Axons arising from the subiculum terminate at the nucleus accumbens, medial prefrontal cortex, and septal regions (Carr and Sesack 1996; Jay et al 1992). These excitatory axons from the subiculum and dopaminergic axons from the ventral tegmental area often terminate in close proximity in the prefrontal cortex (Carr and Sesack 1996) and the nucleus accumbens (Totterdell et al 1989) and can modulate activities in both the prefrontal cortex (Floresco and Grace 2003; Gurden et al 1999) and the nucleus accumbens (Floresco et al 2001; Yang and Mogenson 1986). Moreover, excitatory inputs from the hippocampus (subiculum) to the nucleus accumbens indirectly regulate activity of dopamine neurons (Floresco et al 2001).

The hippocampus, ventral tegmental area, prefrontal cortex, and nucleus accumbens, as well as the mediodorsal thalamus, form a crucial circuit for cognitive function. Projections from hippocampus to prefrontal cortex or nucleus accumbens via the fornix are involved in the modulation of sensorimotor processes (e.g., sensory gating: Bast and Feldon 2003; Pouzet et al 1999 and spatial learning and memory (Brasted et al 2003; Floresco et al 1999; Galani et al 2002; White et al 2003). Functional abnormalities in sensory gating are present in schizophrenia (Ludewig et al 2003; Perry et al 2002); thus the hippocampus-fornix-target zone pathway may be important for understanding schizophrenia.

Hippocampal volume reduction is a consistent finding in schizophrenia neuroimaging studies (McCarley et al 1999; Nelson et al 1998; Shenton et al 2001). Postmortem studies of schizophrenia have reported abnormalities in hippocampus in schizophrenia (Heckers and Konradi 2002), including reduced neuron size (Benes et al 1991) and abnormal cell orientation (Kuroki and Matsushita 1998). Such abnormalities in morphology and cell arrangement may reflect abnormal connectivity in the hippocampal formation, which may in turn result in abnormal hippocampal output and cognitive dysfunction.

In one of the few studies of the fornix in schizophrenia, Chance et al (1999) found significant increased fiber density in the postmortem left fornix in male subjects with schizophrenia, without significant differences in cross-sectional area or total fiber number and concluded that the fornix is not a primary site of neuroanatomic changes in schizophrenia. In magnetic resonance imaging (MRI) studies, Zahajszky et al (2001) found no differences in fornix volume between patients with chronic schizophrenia and normal control subjects but did report a significant correlation between fornix and hippocampus volumes only in the schizophrenic group. In contrast, Davies et al (2001) found a significantly larger cross-sectional area of the fornix in early-onset schizophrenia. Differences in subject characteristics (i.e., early-onset patients, chronic patients, or aged patients) and methods (i.e., conventional MR images are insensitive to subtle abnormalities in the white matter) may contribute to the different structural MRI findings. 
Diffusion tensor imaging (DTI; Basser 1995) affords an opportunity to detect subtle abnormalities in directionally organized tissues such as white matter. More specifically, the axons in white matter are directionally organized along the axis of the bundle, resulting in high diffusion anisotropy, that is, restricted in directions not parallel with the bundle. Mean diffusivity $\left(D_{m}\right)$ is a measure of the average diffusion. In white matter, $D_{m}$ depends on water content and restrictions of water molecule movement. Fractional anisotropy (FA, an index of anisotropy) in white matter depends on several factors, including axon density and coherence (Basser and Jones 2002; Beaulieu 2002). In younger brains, anisotropy increases and mean diffusivity decreases in white matter with increasing age (Neil et al 2002). In healthy older subjects, anisotropy reduces and mean diffusivity increases in white matter with increasing age (Sullivan and Pfefferbaum 2003). Pathological white matter axonal degeneration or demyelination show reduced anisotropy and increased mean diffusivity, as, for example, in multiple sclerosis (Filippi et al 2001), amyotrophic lateral sclerosis (Ellis et al 1999), and Alzheimer's disease (Rose et al 2000). These changes may reflect demyelination, axonal loss, or edema (Beaulieu 2002; Horsfield and Jones 2002). Generally, reduced FA in white matter suggests less coherence of fibers (e.g., crossing fibers) or less dense fibers. In contrast, increased $\mathrm{D}_{\mathrm{m}}$ may suggest immaturity or degeneration in this region. A few studies in schizophrenia report low anisotropy in prefrontal cortex (Buchsbaum et al 1998), in the splenium of the corpus callosum (Agartz et al 2001; Foong et al 2000), or in the whole white matter (Agartz et al 2001; Lim et al 1999; Minami et al 2003). Kubicki et al (2002) found a lack of normal asymmetry in anisotropy in the uncinate fasciculus and lower anisotropy and area of the cingulum bundle (Kubicki et al 2003) in patients with schizophrenia compared with control subjects. Voxel-based methods have reported abnormalities in several areas in patients with schizophrenia (Ardekani et al 2003; Burns et al 2003). There are, however, no DTI studies of the fornix in schizophrenia, most likely because the size of the fornix is near the limit of resolution for reliable measurement of current methods.

The pathology of the fornix in schizophrenia is thus not well understood. In this study, DTI was used to evaluate the fornix. Hippocampal volume was evaluated using high-resolution MR images. Associations between fornix DTI measures and hippocampal structural MRI measures were examined.

\section{Methods and Materials}

\section{Subjects}

Twenty-four male patients with chronic schizophrenia, recruited from inpatient, day treatment, outpatient, and foster care programs at the Veteran's Administration Boston Healthcare System, Brockton, Massachusetts, and 31 male normal control subjects, recruited through newspaper advertisement, participated in the study. Subjects included 21 new subjects (8 patients, 13 control subjects) and 34 subjects ( 16 patients, 18 control subjects) common to our most recently published DTI study of cingulum bundle (Kubicki et al 2003). The research was approved by the local institutional review board. Following a complete description of the study, written informed consent was obtained.

The protocols for diagnosis and clinical evaluations have been previously described (Kubicki et al 2002, 2003; Onitsuka et al 2003). All subjects met the following criteria: aged 18 to 55, IQ greater than 75, right handed (Oldfield 1971), no history of seizures or head trauma with loss of consciousness or a neurological disorder, and no alcohol or drug dependence in the past 5 years. Normal control subjects had no history of Axis I mental disorders in themselves or their first-degree relatives and no Axis II disorder as confirmed by the SCID-NP (Spitzer et al 1990b) and SCID II (Spitzer et al 1990c) by a trained interviewer (MF). The same trained interviewer (MF) diagnosed patients based on the DSM-IV, using the SCID (Spitzer et al 1990a) and a review of medical records. All patients were receiving antipsychotic medication 
when the MR scan was acquired. Five patients were taking typical antipsychotics, 17 patients were taking atypical antipsychotics, and two patients were taking both. All medication dosages were converted to chlorpromazine equivalents (Bezchlibnyk-Butler et al 1996; Stoll 2001).

Socioeconomic status (SES) of patients and control subjects and of their parents (PSES) were evaluated (Hollingshead 1965). Demographic data are shown in Table 1. As part of a comprehensive neuropsychological battery, all subjects were evaluated by the Wechsler Memory Scale-3rd ed. (WMS-III; Wechsler 1997), which includes immediate and delayed measures of verbal and visual memory. Clinical symptoms were measured by the Scale for the Assessment of Positive Symptoms (SAPS; Andreasen 1984) and the Scale for the Assessment of Negative Symptoms (SANS; Andreasen 1981).

\section{MRI Acquisition and Processing}

All subjects underwent line-scan diffusion tensor imaging (LSDI; Gudbjartssen et al 1996; Maier et al 1998) using a quadrature head coil on a 1.5-Tesla GE Echospeed system (General Electric Medical Systems, Milwaukee, Wisconsin), permitting maximum gradient amplitudes of $40 \mathrm{mT} / \mathrm{m}$. Three T1-weighted (T1W) images were used as localizers (sagittal, axial oblique aligned to the anterior commissure-posterior commissure [AC-PC] line, and sagittal oblique aligned to the interhemispheric fissure). From the sagittal oblique T1W image, the doubleoblique LSDI sequence plane was tilted toward the axial plane and rotated about the z-axis resulting in an orientation perpendicular to the AC-PC line. For each line, six images with high $\left(1000 \mathrm{sec} / \mathrm{mm}^{2}\right)$ diffusion-weighting along six noncollinear and noncoplanar directions were collected. For low $\left(5 \mathrm{sec} / \mathrm{mm}^{2}\right)$ diffusion weighting, only two images were collected, because diffusion-related signal changes are minimal. The following scan parameters were used: field of view (FOV) $220 \times 165 \mathrm{~mm}$; scan matrix $128 \times 96$ (image matrix $256 \times 192$ ); slice thickness $=4 \mathrm{~mm}$; interslice distance $=1 \mathrm{~mm}$; receiver bandwidth $\pm 4 \mathrm{kHz}$; echo time $=64 \mathrm{msec}$; effective repetition time $=2592 \mathrm{msec} ;$ scan time $=60 \mathrm{sec} /$ slice. The entire brain was acquired $(31-35$ coronal slices depending on brain size). Reconstructed diffusion-weighted images were transferred to a SUN workstation, where eigenvalues $\left(\lambda_{1}, \lambda_{2}, \lambda_{3}\right)$, eigenvectors (Figure 1), fractional anisotropy (FA), and diffusivity $\left(\mathrm{D}_{\mathrm{m}}\right)$ maps of the diffusion tensor were calculated. FA and $D_{m}$ were calculated using the following formulae (Papadakis et al 1999):

$$
\begin{gathered}
F A=\frac{\sqrt{\left(\lambda_{1}-\lambda_{2}\right)^{2}+\left(\lambda_{2}-\lambda_{3}\right)^{2}+\left(\lambda_{1}-\lambda_{3}\right)^{2}}}{\sqrt{2} \sqrt{\lambda_{1}^{2}+\lambda_{2}{ }^{2}+\lambda_{3}{ }^{2}}} \\
D_{m}=\frac{\lambda_{1}+\lambda_{2}+\lambda_{3}}{3}
\end{gathered}
$$

Movement artifacts were detected and replaced with interpolated data from neighboring line segments. Motion-related artifact maps were also constructed. This measure was defined as the number of line segments missing in the raw LSDI data.

To evaluate hippocampal volume, two additional MRI protocols were executed. A coronal series of contiguous spoiled gradient/recall acquisition (SPGR) images (repetition time $=35$ msec, echo time $=5 \mathrm{msec}, 1$ repetition, $45^{\circ}$ nutation angle, 24-cm field of view, number of excitations $=1.0$, matrix $=256 \times 256[192$ phase encoding steps $] \times 124$, voxel dimensions: . $9375 \times .9375 \times 1.5 \mathrm{~mm}$ ) was used for measuring hippocampus. An axial series of contiguous double-echo (proton density and T2-weighted) images (repetition time $=3000 \mathrm{msec}$, echo time $=30$ and $80 \mathrm{msec}, 24-\mathrm{cm}$ field of view, an interleaved acquisition with 3-mm slice thickness, Voxel dimensions: $.9375 \times .9375 \times 3.0 \mathrm{~mm}$ ) was used to measure total intracranial contents (ICC). Presaturation of a slab inferior to the head was performed for both acquisitions to reduce flow-related artifacts and to obtain low arterial signal intensity. 


\section{DTI Definitions of Fornix}

The body of the fornix is visible as one structure on FA maps, with three to four slices between the anterior commissure and the crux of the fornix. The coronal slice most perpendicular to the fornix was used to evaluate the cross-sectional area of the fornix, reducing partial volume effects. To detect the fornix, a method similar to that of Kubicki et al $(2002,2003)$ was used. A point centered within each fiber tract was selected on the FA coronal map. To extract the fornix from neighboring structures, segmentation was done by thresholding the out-of-plane principal diffusion component (Figure 2), calculated using the following formula.

$\mathrm{L} 1 \mathrm{z}=\lambda_{1} \mathrm{e}_{1 \mathrm{z}}$

L1z: the out-of-plane principal diffusion component

$\lambda_{1}$ : the largest eigenvalue (Figure 1A)

$\mathrm{e}_{1}$ : the eigenvector associated with the largest eigenvalue $\lambda_{1}$ (Figure 1A)

$\mathrm{e}_{1 \mathrm{z}}$ : the out-of-plane component of $\mathrm{e}_{1}$ (Figure 1A and 1B)

We used $1.35 \times 10^{-3} \mathrm{~mm}^{2} / \mathrm{sec}$ as the fixed threshold for all cases (Figure 2).

We also used angular information of $\mathrm{e}_{1}$ for each voxel to separate the fornix from other structures and to remove artifacts. We excluded voxels where the direction of eigenvector $\mathrm{e}_{1}$ deviated more than 20 degrees from the neighboring voxels. We also generated two angular maps for $\mathrm{e}_{1}$ to select the most perpendicular slice of the fornix to the double-oblique coronal plane for each case. Two angular values were calculated at each voxel, that is, an angular deviation $\mathrm{e}_{1}$ from the sagittal plane, which indicated deviation of $\mathrm{x}$ (i.e., in-plane horizontal axis [left-right], Figure 1) component and an angular deviation of $\mathrm{e}_{1}$ from the axial plane, which indicated deviation of y (in-plane vertical axis [superior-inferior], Figure 1) component. The sagittal plane and the axial plane in the present study were orthogonal to the double-oblique coronal plane. These angular values were defined using the following formulas:

$$
\begin{aligned}
& \operatorname{angle}(x)=\operatorname{atan}\left(\frac{\left|e_{1 x}\right|}{\sqrt{e_{1 y^{2}+e_{1 z^{2}}}}}\right) \\
& \operatorname{angle}(y)=\operatorname{atan}\left(\frac{\left|e_{1 y}\right|}{\sqrt{e_{1 x^{2}+e_{1 z^{2}}}}}\right)
\end{aligned}
$$

angle $(x)$ : the angle between $\mathrm{e}_{1}$ and the sagittal plane

angle $(y)$ : the angle between $\mathrm{e}_{1}$ and the axial plane $\mathrm{e}_{1}=\left(\mathrm{e}_{1 \mathrm{x}}, \mathrm{e}_{1 \mathrm{y}}, \mathrm{e}_{1 \mathrm{z}}\right)$

$e_{1 x}$ : the in-plane horizontal component of $\mathrm{e}_{1}$ (Figure 1A and 1B)

$e_{1 y}$ : the in-plane vertical component of $\mathrm{e}_{1}$ (Figure $1 \mathrm{~A}$ and $1 \mathrm{~B}$ )

$e_{1 z}$ : the out-of-plane component of $\mathrm{e}_{1}$ (Figure 1A and 1B)

We selected the slice with the lowest value of mean magnitude of two angles, averaged in the delineated fornix over each slice (Figure 2). Cross-sectional area of the fornix was calculated for this slice.

To calculate the mean FA and $\mathrm{D}_{\mathrm{m}}$, we used three slices, including the slice just anterior to the selected slice, the selected slice, and the slice just posterior to the selected slice. To avoid potential effects of low FA values in the surrounding area due to partial volume effects, we selected 16 voxels with the highest FA values in delineated fornix, usually located at the center of the fornix for each slice. The mean FA and $\mathrm{D}_{\mathrm{m}}$ of these 48 voxels were calculated. 


\section{Definition of Hippocampus on Structural MRI Scans Using Anatomic Landmarks}

The hippocampus was manually delineated blind to diagnosis and included Ammon's horn, dentate gyrus, and subiculum. Using criteria similar to Pantel et al (2000), the head of the hippocampus was separated from the amygdala in the sagittal plane. The fimbria and alveus, white matter in the hippocampus visible on SPGR images, were used to separate the hippocampus from the amygdala rostrally and choroid plexus caudally. The posterior border of the hippocampus was defined as the last slice showing the fornix along the crux of the hippocampus. All editing was confirmed on sagittal, axial, and coronal planes. Interrater reliability for the hippocampus was evaluated by three independent raters (NK, JL, SW) on 10 randomly selected cases. Intraclass correlation coefficients were .95 for the left hippocampus and .93 for the right hippocampus.

\section{Statistical Analysis}

Mann-Whitney's $U$ tests were performed to assess group differences in age, SES, parental SES, IQ, handedness, and between the groups of subjects taking typical and atypical antipsychotics with medication dosage converted to chlorpromazine equivalents. Fisher's Exact Tests were performed to assess group difference in a past history of alcohol abuse or other substance abuse.

The Shapiro-Wilk test of normality demonstrated normal distributions $(p>.10)$ for all MR measures, including FA values, mean $\mathrm{D}_{\mathrm{m}}$ values, cross-sectional area of the fornix, and hippocampal volumes. Student's $t$ tests were performed to assess group differences in the number of missing line segments due to movement artifacts. The statistical conclusions reported later remained the same when we performed analyses of covariance (ANCOVAs), using one between-subjects factor (group: schizophrenia patients or control subjects) and one covariate (age).

To test for group differences in ICC, a Student's $t$ test was performed. For comparison of the hippocampal volume, a two-factor analysis of variance (ANOVA) was performed, using one between-subjects factor (group: schizophrenia patients or control subjects) and one withinsubjects factor (hemisphere: left or right). Absolute volume and relative volume (region of interest [ROI] volume/ICC volume) were separately used as the dependent measure. Of note, the statistical conclusions reported below remained the same when absolute volume was analyzed with ICC as a covariate. Moreover, statistical conclusions also remained the same when a two-factor ANCOVA was performed, using one covariate (age). The same analyses were performed to assess differences between groups of subjects taking typical and atypical antipsychotics.

To evaluate correlations between DTI measures of fornix and hippocampal volumes Pearson's Product-Moment correlation coefficients were calculated. Absolute volumes were used for hippocampal volumes. Due to statistically significant correlations among mean FA, crosssectional area of the fornix, and medication dosage, we also computed partial correlation coefficients to control for medication.

Exploratory analyses of the relationship between MRI measures and clinical measures (i.e., age, age at onset, duration of illness, medication dosage, four SAPS and five SANS subscores, total scores for SAPS and SANS, IQ, and the score for "general memory" of WMS-III) were computed using Spearman's rho. Because these analyses were exploratory in nature, we used $p<.05$ for statistical significance, rather than correcting for multiple correlations. We also computed partial correlation coefficients controlling for medication. 


\section{Results}

There were no significant group differences in age or PSES. Patients with schizophrenia showed significantly lower SES and IQ than control subjects (Table 1). There were no significant group differences in history of alcohol abuse or other substance abuse $(p>.1)$. There were no significant differences between groups taking typical and atypical antipsychotics in medication dosages $(t[20]=-.454, p=.654)$. There were no significant group differences in ICC volume.

\section{Measures of the Fornix}

Schizophrenic patients had lower FA (7.5\% less, $t[53]=-2.963, p=.005$, Table 2, Figure 3 ), and higher $\mathrm{D}_{\mathrm{m}}$ in the fornix compared with control subjects $(6.7 \%$ more, $t[53]=-2.096, p=$. 041, Table 2, Figure 3). Schizophrenic patients also showed a smaller cross-sectional area of the fornix than control subjects (15.5\% smaller, $t[53]=-3.265, p=.002$, Table 2, Figures 3 and 4). There were no statistically significant differences between patient groups taking typical versus atypical antipsychotics in any fornix MR measure.

For movement artifacts, there were no significant differences in the number of missing line segments between two groups $(t[53]=-.122, p=.904)$.

Data and statistical analyses are shown in Table 2 .

\section{Comparison of Hippocampal Volume}

Comparison of relative hippocampal volume (ROI volume/ICC volume) revealed significant group differences $[F(1,53)=9.557, p=.003]$. There was also a significant main effect of hemisphere $[F(1,53)=7.422, p=.009]$ and no significant group-by-hemisphere interaction $[F(1,53)=.004, p=.950]$. Patients with schizophrenia showed a reduced volume of $8.8 \%$ (left: $9.0 \%$, right: $8.7 \%$, Table 2, Figure 3) in the hippocampus compared to normal control subjects. Comparison of absolute hippocampal volume also revealed significant group differences $[F(1,53)=15.355, p<.001]$. There were no significant differences in hippocampal volumes between patients taking typical versus atypical antipsychotics.

Data and statistical analyses are shown in Table 2.

\section{Correlations Between Measures of the Fornix and Hippocampal Volumes}

Mean $\mathrm{D}_{\mathrm{m}}$ of the fornix negatively correlated with both left hippocampal volume $(r=-.437$, $p=.033$, partial correlation coefficients controlling for medication dosage: $r=-.423, p=$.

$044)$, and right hippocampal volume $(r=-.587, p=.003$, partial correlation coefficients: $r=$ $-.576, p=.004$ ) for the schizophrenia group only (Figure 5). The cross-sectional area of the fornix positively correlated with both left hippocampal volume $(r=.457, p=.025$, partial correlation coefficients: $r=.450, p=.031)$ and right hippocampal volume $(r=.477, p=.018$, partial correlation coefficients: $r=.453, p=.030$ ) for the schizophrenic group only (Figure 5). FA was not correlated with hippocampal volumes. Hippocampal volumes were not correlated with any measures of the fornix in the control group.

\section{Correlation of MR Measures of the Fornix and the Hippocampus with Clinical Measures}

Age was positively correlated with mean $\mathrm{D}_{\mathrm{m}}(\mathrm{rho}=.403, p=.025)$ of the fornix for the control group. This is similar to findings reporting an increase in mean diffusivity within other regions of the brain in normal aging (Abe et al 2002; Moseley 2002). IQ was not statistically significantly correlated with any MRI measures for either group. In the schizophrenic group, age, age at onset of illness, and duration of illness were not correlated with any MRI measures of the fornix or the hippocampus for the schizophrenic group. Medication dosage was 
negatively correlated with mean FA (rho $=-.679, p<.001$ ) and cross-sectional area of the fornix (rho $=-.477, p=.018)$ and was positively correlated with mean $\mathrm{D}_{\mathrm{m}}($ rho $=.525, p=$. 009).

Cross-sectional area of the fornix was negatively correlated with the SANS score for global attention (rho $=-.520, p=.016$ ), one of five SANS subscores, as reduced cross-sectional area of the fornix correlated with worse SANS rating of global attention. The correlation did not remain significant when controlling for medication dosage (partial correlation coefficients: $r$ $=.351, p=.129)$. In contrast, for neuropsychologic measures of memory, reduced scores on the WMS-III general memory (delayed memory for both verbal and visual material) correlated with reduced left (rho $=.524, p=.018$, partial correlation coefficients controlling for medication dosage: $r=.600, p=.007$, Figure 6 ) and right (rho $=.477, p=.034$, partial correlation coefficients: $r=.590, p=.008$, Figure 6) hippocampal volumes, and these correlations remained significant even when controlling for medication dosage. There were no such correlations for the control group.

\section{Discussion}

To our knowledge, this is the first study to evaluate the fornix specifically using DTI methods. In this study, fiber integrity of the fornix and hippocampal volume were measured in patients with chronic schizophrenia and in normal control subjects. The results showed reduced FA and cross-sectional area and increased $\mathrm{D}_{\mathrm{m}}$ in the fornix in the schizophrenia patients compared with normal control subjects. Additionally, bilateral hippocampal volume was reduced in patients with schizophrenia compared with normal control subjects. Moreover, reduced hippocampal volume was correlated with increased $\mathrm{D}_{\mathrm{m}}$ and reduced cross-sectional area of the fornix in the schizophrenic group.

As discussed previously, FA and $\mathrm{D}_{\mathrm{m}}$ are observed to change as a function of normal aging processes and neurodegenerative processes. Reduced FA and simultaneous increased $\mathrm{D}_{\mathrm{m}}$ indicate that fornix abnormalities may be due to either immaturity or degeneration of the fiber tract. The findings that patients with chronic schizophrenia have abnormalities in the integrity of the fornix indicate abnormal connectivity between the hippocampus and other brain regions. These abnormalities may reflect decreased axonal density, axonal damage, or decreased degree of myelination (Beaulieu 2002). In a postmortem study, Chance et al (1999) found high fiber density in the left fornix in male schizophrenia patients, without significant differences in the total number of fibers. They also measured fiber density in different parts of the fornix (measured in left and right columns of the fornix, which is located superior to the anterior commissure, whereas we measured fornix integrity in the body of the fornix), and their subjects were older (male schizophrenia patients: 62.1 years, male control subjects: 67.3 years) than our subjects. These differences may contribute to inconsistencies in the findings.

Kuzniecky et al (1999) found fornix atrophy in $86 \%$ of temporal lobe epilepsy patients with unilateral hippocampal atrophy and in almost all patients with bilateral symmetrical hippocampal atrophy. This finding suggests that hippocampal atrophy may cause secondary fornix atrophy. Disrupted fornix integrity and reduced cross-sectional area of the fornix in our study may thus reflect abnormal hippocampi in schizophrenia. Of note, the bilateral hippocampal volume reductions and positive correlation between cross-sectional area of the fornix and hippocampal volume in our patients with schizophrenia support this interpretation.

In our study, patients with schizophrenia showed a statistically significantly lower IQ than normal control subjects; however, IQ was not significantly correlated with any MRI measures for either group. Nonetheless, the question of IQ and disease-related structural changes, as revealed by MRI and DTI, requires further study. 
Furthermore, reduced volume of the hippocampus and a disruption in connectivity between the hippocampus and other brain regions may result in dysfunction in important neural circuits, such as between prefrontal cortex, striatum, and thalamus. The correlations between hippocampal volumes and scores on the WMS-III and between the cross-sectional area of the fornix and a measure of negative symptoms (the global attention SANS subscore) are consistent with this disruption hypothesis. Our prior findings revealed evidence of a double dissociation of left uncinate fasciculus and cingulate bundle with deficits in declarative episodic memory and executive function, respectively (Nestor et al 2004). Our current findings add to these data by linking disease-related abnormalities of the fornix and the hippocampus to reduced declarative memory. Taken together, the current and past findings help to demarcate the distributed nature of the functional neuroanatomy of memory disturbance in schizophrenia.

Because this patient population was on antipsychotic mediation, it is not known to what degree the illness itself and chronic antipsychotic treatment contribute to this pathology. There was a correlation between higher dose of antipsychotic medication and reduced mean FA, reduced cross-sectional area of the fornix, and increased mean $\mathrm{D}_{\mathrm{m}}$. Antipsychotic medication may thus affect both fornix integrity and the size of the fornix. There are only a small number of studies that have evaluated the relationship between antipsychotic medication and diffusion measures. Buchsbaum and coworkers (1998) did not find statistically significant differences in FA in the prefrontal cortex between groups receiving antipsychotic treatment for the first time and those patients receiving chronic antipsychotic treatment. Foong et al (2000) also did not find a statistically significant correlation between medication dosage and FA or $\mathrm{D}_{\mathrm{m}}$ in the corpus callosum. Minami et al (2003), however, found a statistically significant correlation between higher FA of left frontal white matter and higher dose of antipsychotic medication. Thus, the effect of antipsychotic medication on DTI measures is still not well understood. On the other hand, there have been several studies of association between antipsychotics and brain morphology. For example, Selemon et al (1999) reported increased volume and astroglial density in prefrontal cortex of monkey brain after 6 months medication for both typical and atypical antipsychotics. It has been suggested that astroglial proliferation may occur in white matter as well, but this is not well documented.

In human studies evaluating the association between white matter morphology and antipsychotics, Christensen et al (2004) found nonsignificant increased white matter volume in schizophrenia patients who failed to respond to antipsychotics after 4 weeks medication but a statistically significant decrease in white matter volume in responders. There was no relationship between receiving medication and white matter changes, and they found no evidence that antipsychotic medication itself caused volumetric change in cerebral white matter. Lieberman et al (2005) also found no significant changes in white matter volume in patients with first-episode psychosis during 104 weeks of receiving either olanzapine or haloperidol. The effect of antipsychotic medication on white matter is thus still not well understood, and a longitudinal study of first-episode patients will be needed to clarify this issue. Whether abnormalities in the integrity of the fornix and the hippocampus are progressive also requires further study. Of note, Davies et al (2001) reported an increase in cross-sectional area of the fornix in patients with early-onset schizophrenia. This suggests that the cross-sectional area of the fornix may be reduced in the postonset course of the illness, although the methodology for measuring cross-sectional area of the fornix, using conventional MRI differs among studies. Other explanations are, however, possible, and further studies are required to know whether illness itself or antipsychotic medication contribute to our findings.

There are several limitations in this study. First, subject populations included men only. Second, we do not know whether the group differences in MR measures reflect effects of diagnosis per se or secondary effects of having a chronic illness such as lack of environmental stimulation, being unemployed, or disabling effects of normative aging processes, although 
duration of illness was not significantly correlated with any MR measures in the schizophrenic group. Moreover, age was correlated with only one MR measure, mean $\mathrm{D}_{\mathrm{m}}$ for control subjects (rho $=-.43, p=.025$ ), but when we covaried for age, all group differences remained the same. Third, we selected three slices to evaluate the integrity of the fornix. We could not, however, evaluate abnormalities in specific regions of the fornix (i.e., left and right columns and crux of fornix, the precommissural fornix) because peripheral parts of the fornix are too small to evaluate reliably using our acquisition method. A better approach for future studies might be to use fiber tractography methods to evaluate the whole fornix. This method is still in development, however. We also note that our LSDI sequence has relatively higher in-plane resolution than out-of-plane resolution, thus making it better for ROI measurement of fiber tracts perpendicular to the coronal (acquisition) plane. Moreover, we used angular information to select the most perpendicular slice of the fornix to the coronal plane. Such a choice should reduce potential effects of partial volumes despite relatively low out-of-plane resolution. Fourth, because of this choice, we were more likely to evaluate slightly different parts of the fornix across subjects. An alternative way to measure the fornix might be to use a predetermined distance along the fornix; however, the fact that the body of fornix has no branches may reduce variation of our measurements. We believe that it should have more advantages to select the most perpendicular slice of the fornix first to reduce potential effects of partial volumes as mentioned earlier. Another alternative way to perform diffusion measurements within the fornix would be to define the ROI on one of the high-resolution structural images and then transfer these coordinates to the DTI image. Small errors of manual segmentation of the fornix and misregistration for transforming these segmentations to DTI images could, however, have significant effects on DTI measures because of the small size of the fornix. We believe that our method, which is to select a reliable portion of the fornix on the DTI image, has more advantages.

In conclusion, abnormal fornix integrity is present in patients with chronic schizophrenia. This abnormality was correlated with bilateral hippocampal volume reduction. In addition, for the patients only, reduced neuropsychologic scores on standardized tests of declarative memory correlated with reduced hippocampal volumes (possibly related more to the entorhinal than the fornix efferent pathway). Our findings strongly suggest that hippocampal dysfunction and disrupted connectivity between the hippocampus and other brain regions may play an important role in both the pathology of schizophrenia as well as in the core neuropsychologic disturbance of the disease. Further studies are nonetheless needed to determine what contributes to fornix and hippocampal abnormalities in schizophrenia, and whether or not they are progressive.

\section{Acknowledgments}

This study was supported, in part, by the Department of Veterans Affairs Merit Awards (MES, RWM), a REAP award (RWM, MES), a Middleton Award (RWM) from the Department of Veterans Affairs, grants from the National Institute of Health (K02 MH 01110 and R01 MH 50747 to MES, RO1 MH 40799 to RWM, and RO3 MH068464-01 to MK), the MIND Institute (Albuquerque, NM, RWM), the National Alliance for Research on Schizophrenia and Depression (MK), the National Alliance for Medical Image Computing (NAMIC), funded by the National Institutes of Health through the NIH Roadmap for Medical Research, Grant U54 EB005149 (MES) and the Welfide Medicinal Research Foundation (Japan, NK).

We gratefully acknowledge the administrative support of Marie Fairbanks and the research assistant support of Erin Conner.

\section{References}

Abe O, Aoki S, Hayashi N, Yamada H, Kunimatsu A, Mori H, et al. Normal aging in the central nervous system: Quantitative MR diffusion-tensor analysis. Neurobiol Aging 2002;23:433-441. [PubMed: 11959406] 
Agartz I, Andersson JL, Skare S. Abnormal brain white matter in schizophrenia: A diffusion tensor imaging study. Neuroreport 2001;12:2251-2254. [PubMed: 11447344]

Andreasen, NC. Scale for the Assessment of Negative Symptoms (SANS). Department of Psychiatry, University of Iowa College of Medicine; Iowa City, IA: 1981.

Andreasen, NC. Scale for the Assessment of Positive Symptoms (SAPS). Department of Psychiatry, University of Iowa College of Medicine; Iowa City, IA: 1984.

Ardekani BA, Nierenberg J, Hoptman MJ, Javitt DC, Lim KO. MRI study of white matter diffusion anisotropy in schizophrenia. Neuroreport 2003;14:2025-2029. [PubMed: 14600491]

Basser PJ. Inferring microstructural features and the physiological state of tissues from diffusionweighted images. NMR Biomed 1995;8:333-344. [PubMed: 8739270]

Basser PJ, Jones DK. Diffusion-tensor MRI: Theory, experimental design and data analysis—a technical review. NMR Biomed 2002;15:456-467. [PubMed: 12489095]

Bast T, Feldon J. Hippocampal modulation of sensorimotor processes. Prog Neurobiol 2003;70:319-345. [PubMed: 12963091]

Beaulieu C. The basis of anisotropic water diffusion in the nervous system-a technical review. NMR Biomed 2002;15:435-455. [PubMed: 12489094]

Benes FM. Evidence for altered trisynaptic circuitry in schizophrenic hippocampus. Biol Psychiatry 1999;46:589-599. [PubMed: 10472413]

Benes FM, Sorensen I, Bird ED. Reduced neuronal size in posterior hippocampus of schizophrenic patients. Schizophr Bull 1991;17:597-608. [PubMed: 1805353]

Bezchlibnyk-Butler, KZ.; Jeffries, JJ. Clinical Handbook of Psychotropic Drugs. Vol. 5th rev. ed.. Hogrefe and Huber; Seattle: 1996.

Brasted PJ, Bussey TJ, Murray EA, Wise SP. Role of the hippocampal system in associative learning beyond the spatial domain. Brain 2003;126:1202-1223. [PubMed: 12690059]

Buchsbaum MS, Tang CY, Peled S, Gudbjartsson H, Lu D, Hazlett EA, et al. MRI white matter diffusion anisotropy and PET metabolic rate in schizophrenia. Neuroreport 1998;9:425-430. [PubMed: 9512384]

Burns J, Job D, Bastin ME, Whalley H, Macgillivray T, Johnstone EC, et al. Structural disconnectivity in schizophrenia: A diffusion tensor magnetic resonance imaging study. Br J Psychiatry 2003;182:439-443. [PubMed: 12724248]

Carr DB, Sesack SR. Hippocampal afferents to the rat prefrontal cortex: Synaptic targets and relation to dopamine terminals. J Comp Neurol 1996;369:1-15. [PubMed: 8723699]

Chance SA, Highley JR, Esiri MM, Crow TJ. Fiber content of the fornix in schizophrenia: Lack of evidence for a primary limbic encephalopathy. Am J Psychiatry 1999;156:1720-1724. [PubMed: 10553734]

Christensen J, Holcomb J, Garver DL. State-related changes in cerebral white matter may underlie psychosis exacerbation. Psychiatry Res 2004;130:71-78. [PubMed: 14972369]

Davies DC, Wardell AM, Woolsey R, James AC. Enlargement of the fornix in early-onset schizophrenia: A quantitative MRI study. Neurosci Lett 2001;301:163-166. [PubMed: 11257423]

Ellis CM, Simmons A, Jones DK, Bland J, Dawson JM, Horsfield MA, et al. Diffusion tensor MRI assesses corticospinal tract damage in ALS. Neurology 1999;53:1051-1058. [PubMed: 10496265]

Filippi M, Cercignani M, Inglese M, Horsfield MA, Comi G. Diffusion tensor magnetic resonance imaging in multiple sclerosis. Neurology 2001;56:304-311. [PubMed: 11171893]

Floresco SB, Blaha CD, Yang CR, Phillips AG. Modulation of hippocampal and amygdalar-evoked activity of nucleus accumbens neurons by dopamine: Cellular mechanisms of input selection. $\mathrm{J}$ Neurosci 2001;21:2851-2860. [PubMed: 11306637]

Floresco SB, Braaksma DN, Phillips AG. Thalamic-cortical-striatal circuitry subserves working memory during delayed responding on a radial arm maze. J Neurosci 1999;19:11061-11071. [PubMed: 10594086]

Floresco SB, Grace AA. Gating of hippocampal-evoked activity in prefrontal cortical neurons by inputs from the mediodorsal thalamus and ventral tegmental area. J Neurosci 2003;23:3930-3943.

[PubMed: 12736363] 
Floresco SB, Todd CL, Grace AA. Glutamatergic afferents from the hippocampus to the nucleus accumbens regulate activity of ventral tegmental area dopamine neurons. J Neurosci 2001;21:49154922. [PubMed: 11425919]

Foong J, Maier M, Clark CA, Barker GJ, Miller DH, Ron MA. Neuropathological abnormalities of the corpus callosum in schizophrenia: A diffusion tensor imaging study. J Neurol Neurosurg Psychiatry 2000;68:242-244. [PubMed: 10644799]

Galani R, Obis S, Coutureau E, Jarrard L, Cassel JC. A comparison of the effects of fimbria-fornix, hippocampal, or entorhinal cortex lesions on spatial reference and working memory in rats: short versus long postsurgical recovery period. Neurobiol Learn Mem 2002;77:1-16. [PubMed: 11749082]

Gudbjartsson H, Maier SE, Mulkern RV, Morocz IA, Patz S, Jolesz FA. Line scan diffusion imaging. Magn Reson Med 1996;36:509-519. [PubMed: 8892201]

Gurden H, Tassin JP, Jay TM. Integrity of the mesocortical dopaminergic system is necessary for complete expression of in vivo hippocampal-prefrontal cortex long-term potentiation. Neuroscience 1999;94:1019-1027. [PubMed: 10625044]

Heckers S, Konradi C. Hippocampal neurons in schizophrenia. J Neural Transm 2002;109:891-905. [PubMed: 12111476]

Hollingshead, AB. Two Factor Index of Social Position. Yale University Press; New Haven, CT: 1965.

Horsfield MA, Jones DK. Applications of diffusion-weighted and diffusion tensor MRI to white matter diseases—a review. NMR Biomed 2002;15:570-577. [PubMed: 12489103]

Jay TM, Thierry AM, Wiklund L, Glowinski J. Excitatory amino acid pathway from the hippocampus to the prefrontal cortex. Contribution of AMPA receptors in hippocampo-prefrontal cortex transmission. Eur J Neurosci 1992;4:1285-1295. [PubMed: 12106392]

Kubicki M, Westin CF, Maier SE, Frumin M, Nestor PG, Salisbury DF, et al. Uncinate fasciculus findings in schizophrenia: A magnetic resonance diffusion tensor imaging study. Am J Psychiatry 2002;159:813-820. [PubMed: 11986136]

Kubicki M, Westin CF, Nestor PG, Wible CG, Frumin M, Maier SE, et al. Cingulate fasciculus integrity disruption in schizophrenia: A magnetic resonance diffusion tensor imaging study. Biol Psychiatry 2003;54:1171-1180. [PubMed: 14643084]

Kuroki N, Matsushita M. Pyramidal cell orientation and density in the hippocampus in schizophrenia. Neuropathology 1998;18:235-241.

Kuzniecky R, Bilir E, Gilliam F, Faught E, Martin R, Hugg J. Quantitative MRI in temporal lobe epilepsy: Evidence for fornix atrophy. Neurology 1999;53:496-501. [PubMed: 10449110]

Lieberman JA, Tollefson GD, Charles C, Zipursky R, Sharma T, Kahn RS, et al. Antipsychotic drug effects on brain morphology in first-episode psychosis. Arch Gen Psychiatry 2005;62:361-370. [PubMed: 15809403]

Lim KO, Hedehus M, Moseley M, de Crespigny A, Sullivan EV, Pfefferbaum A. Compromised white matter tract integrity in schizophrenia inferred from diffusion tensor imaging. Arch Gen Psychiatry 1999;56:367-374. [PubMed: 10197834]

Ludewig K, Geyer MA, Vollenweider FX. Deficits in prepulse inhibition and habituation in nevermedicated, first-episode schizophrenia. Biol Psychiatry 2003;54:121-128. [PubMed: 12873801]

Maier SE, Gudbjartsson H, Patz S, Hsu L, Lovblad KO, Edelman RR, et al. Line scan diffusion imaging: Characterization in healthy subjects and stroke patients. AJR Am J Roentgenol 1998;171:85-93. [PubMed: 9648769]

McCarley RW, Wible CG, Frumin M, Hirayasu Y, Levitt JJ, Fischer IA, et al. MRI anatomy of schizophrenia. Biol Psychiatry 1999;45:1099-1119. [PubMed: 10331102]

Minami T, Nobuhara K, Okugawa G, Takase K, Yoshida T, Sawada S, et al. Diffusion tensor magnetic resonance imaging of disruption of regional white matter in schizophrenia. Neuropsychobiology 2003;47:141-145. [PubMed: 12759557]

Moseley M. Diffusion tensor imaging and aging — a review. NMR Biomed 2002;15:553-560. [PubMed: 12489101]

Neil J, Miller J, Mukherjee P, Huppi PS. Diffusion tensor imaging of normal and injured developing human brain—a technical review. NMR Biomed 2002;15:543-552. [PubMed: 12489100] 
Nelson MD, Saykin AJ, Flashman LA, Riordan HJ. Hippocampal volume reduction in schizophrenia as assessed by magnetic resonance imaging: A meta-analytic study. Arch Gen Psychiatry 1998;55:433440. [PubMed: 9596046]

Nestor PG, Kubicki M, Gurrera RJ, Niznikiewicz M, Frumin M, McCarley RW, et al. Neuropsychological correlates of diffusion tensor imaging in schizophrenia. Neuropsychology 2004;18:629-637. [PubMed: 15506830]

Oldfield RC. The assessment and analysis of handedness: The Edinburgh inventory. Neuropsychologia 1971;9:97-113. [PubMed: 5146491]

Onitsuka T, Shenton ME, Kasai K, Nestor PG, Toner SK, Kikinis R, et al. Fusiform gyrus volume reduction and facial recognition in chronic schizophrenia. Arch Gen Psychiatry 2003;60:349-355. [PubMed: 12695311]

Pantel J, O'Leary DS, Cretsinger K, Bockholt HJ, Keefe H, Magnotta VA, et al. A new method for the in vivo volumetric measurement of the human hippocampus with high neuroanatomical accuracy. Hippocampus 2000;10:752-758. [PubMed: 11153720]

Papadakis NG, Xing D, Houston GC, Smith JM, Smith MI, James MF, et al. A study of rotationally invariant and symmetric indices of diffusion anisotropy. Magn Reson Imaging 1999;17:881-892. [PubMed: 10402595]

Perry W, Feifel D, Minassian A, Bhattacharjie I, Braff DL. Information processing deficits in acutely psychotic schizophrenia patients medicated and unmedicated at the time of admission. Am J Psychiatry 2002;159:1375-1381. [PubMed: 12153831]

Pouzet B, Feldon J, Veenman CL, Yee BK, Richmond M, Nicholas J, et al. The effects of hippocampal and fimbria-fornix lesions on prepulse inhibition. Behav Neurosci 1999;113:968-981. [PubMed: 10571479]

Rose SE, Chen F, Chalk JB, Zelaya FO, Strugnell WE, Benson M, et al. Loss of connectivity in Alzheimer's disease: An evaluation of white matter tract integrity with colour coded MR diffusion tensor imaging. J Neurol Neurosurg Psychiatry 2000;69:528-530. [PubMed: 10990518]

Schmajuk NA. Hippocampal dysfunction in schizophrenia. Hippocampus 2001;11:599-613. [PubMed: 11732711]

Selemon LD, Lidow MS, Goldman-Rakic PS. Increased volume and glial density in primate prefrontal cortex associated with chronic antipsychotic drug exposure. Biol Psychiatry 1999;46:161-172. [PubMed: 10418690]

Shenton ME, Dickey CC, Frumin M, McCarley RW. A review of MRI findings in schizophrenia. Schizophr Res 2001;49:1-52. [PubMed: 11343862]

Spitzer, RL.; Williams, JBW.; Gibbson, M.; First, M. The Structured Clinical Interview for DSM-III-R (SCID). American Psychiatric Association; Washington, DC: 1990a.

Spitzer, RL.; Williams, JBW.; Gibbson, M.; First, M. The Structured Clinical Interview for DSM-III-RNon-Patient Edition (SCID-NP). American Psychiatric Association; Washington, DC: 1990b.

Spitzer, RL.; Williams, JBW.; Gibbson, M.; First, M. The Structured Clinical Interview for DSM-III-RPersonality-Disorder (SCID-II). American Psychiatric Association; Washington, DC: 1990c.

Stoll, AL. The Psychopharmacology Reference Card: Antipsychotic Treatment Guide. McLean Hospital; Belmont, MA: 2001.

Sullivan EV, Pfefferbaum A. Diffusion tensor imaging in normal aging and neuropsychiatric disorders. Eur J Radiol 2003;45:244-255. [PubMed: 12595109]

Totterdell S, Smith AD. Convergence of hippocampal and dopaminergic input onto identified neurons in the nucleus accumbens of the rat. J Chem Neuroanat 1989;2:285-298. [PubMed: 2572241]

Wechsler, D. The Wechsler Memory Scale. Vol. 3rd edition. Psychological Corp: Harcourt Brace Jovanovich; San Antonio, TX: 1997.

Weinberger DR. Cell biology of the hippocampal formation in schizophrenia. Biol Psychiatry 1999;45:395-402. [PubMed: 10071707]

White NM, Holahan MR, Goffaux P. Involuntary, unreinforced (pure) spatial learning is impaired by fimbria-fornix but not by dorsal hippocampus lesions. Hippocampus 2003;13:324-333. [PubMed: 12722973] 
Yang CR, Mogenson GJ. Dopamine enhances terminal excitability of hippocampal-accumbens neurons via D2 receptor: Role of dopamine in presynaptic inhibition. J Neurosci 1986;6:2470-2478. [PubMed: 3528409]

Zahajszky J, Dickey CC, McCarley RW, Fischer IA, Nestor P, Kikinis R, et al. A quantitative MR measure of the fornix in schizophrenia. Schizophr Res 2001;47:87-97. [PubMed: 11163548] 
A

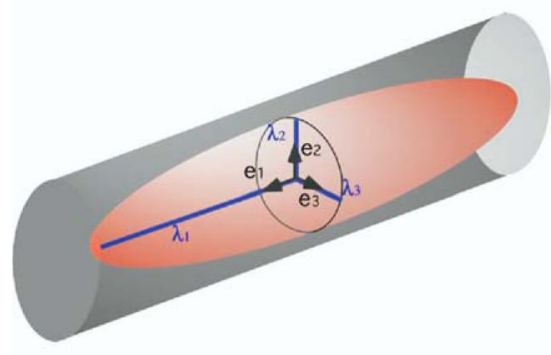

B

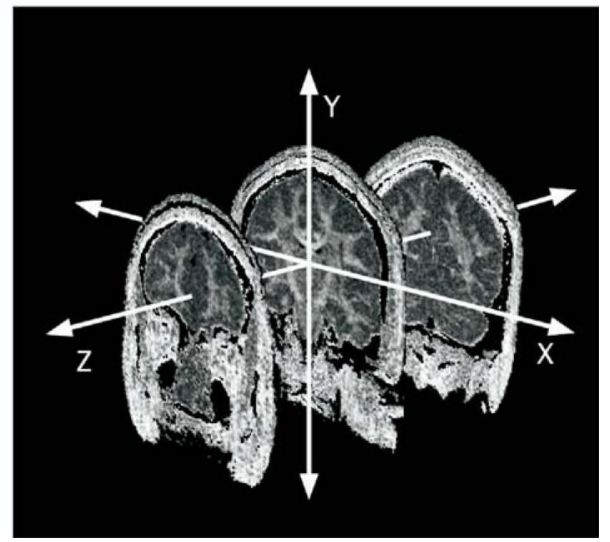

Figure 1.

(A) An illustration showing anisotropic diffusion. In directionally well-organized tissue, such as the white-matter fiber bundles, diffusion along one direction is greater than the others (i.e., $\left.\lambda_{1}>>\lambda_{2}>\lambda_{3}\right) . \lambda 1$ : the largest eigenvalue, which corresponds to the length of the long radial of the ellipsoid; $\lambda_{2}$ : the second eigenvalue; $\lambda_{3}$ : the smallest eigenvalue; $\mathrm{e}_{1}$ : the eigenvector with the largest eigenvalue; $e_{2}$ : the eigenvector with the second eigenvalue; $\mathrm{e}_{3}$ : the eigenvector with the smallest eigenvalue. (B) An illustration showing the $x-y-z$ coordinate system that we used. Images are FA maps. $\mathrm{x}$ : in-plane horizontal axis (left-right), $\mathrm{y}$ : in-plane vertical axis (superiorinferior), z: out-of-plane axis (anterior-posterior). 
FA Map
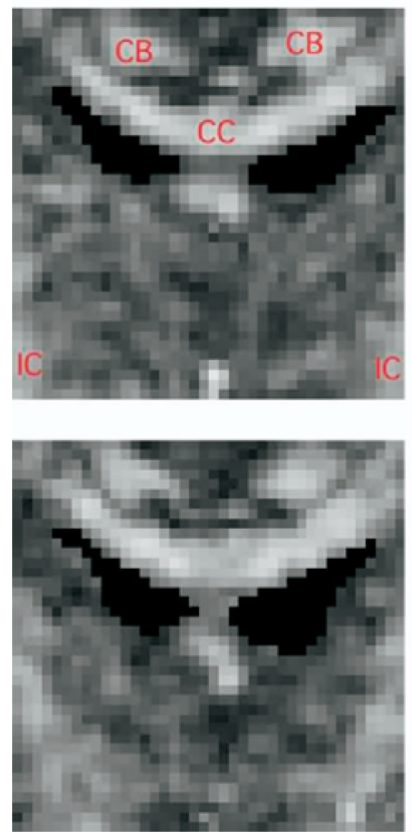

Out-of-Plane Principal Diffusion Component Map
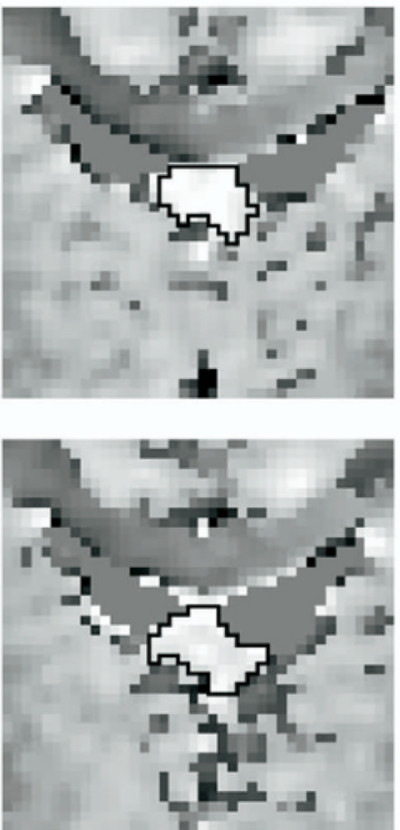

Color-coded map
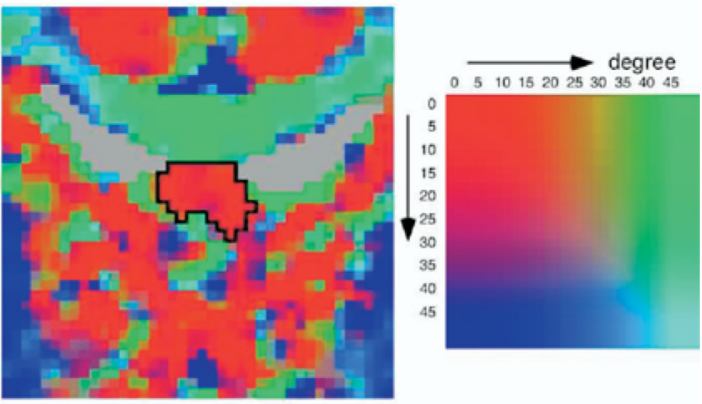

Figure 2.

Fractional anisotropy map, out-of-plane principal diffusion component map, and color-coded map. Upper row: the selected slice. Lower row: the slice just anterior to the selected slice. The boundary of fornix is shown in the out-of-plane principal diffusion component maps and the color-coded maps. In out-of-plane principal diffusion component map, high-intensity corresponds to the fiber tract perpendicular to the plane, and the fornix is easy to differentiate. Two angle maps were merged in this color-coded map to make it more intuitively understandable to the reader. One is a map for the angle between the first eigenvector and the sagittal plane. The other is a map for the angle between the first eigenvector and the axial plane. The color table shows these two angular values of each color. Red color means the first eigenvector closely perpendicular to the coronal plane. Green color means the eigenvector with medial-lateral direction. Green color in the superior part of each color-coded image corresponds to the corpus callosum. Blue color means the eigenvector with superior-inferior direction. Blue color in the inferior part of both sides corresponds to the internal capsule. Blue color in the delineated fornix of the slice just anterior to the selected slice indicates that the fornix is descending inferiorly in this slice. $\mathrm{CB}$, cingulum bundle; $\mathrm{CC}$, corpus callosum; IC, internal capsule. 

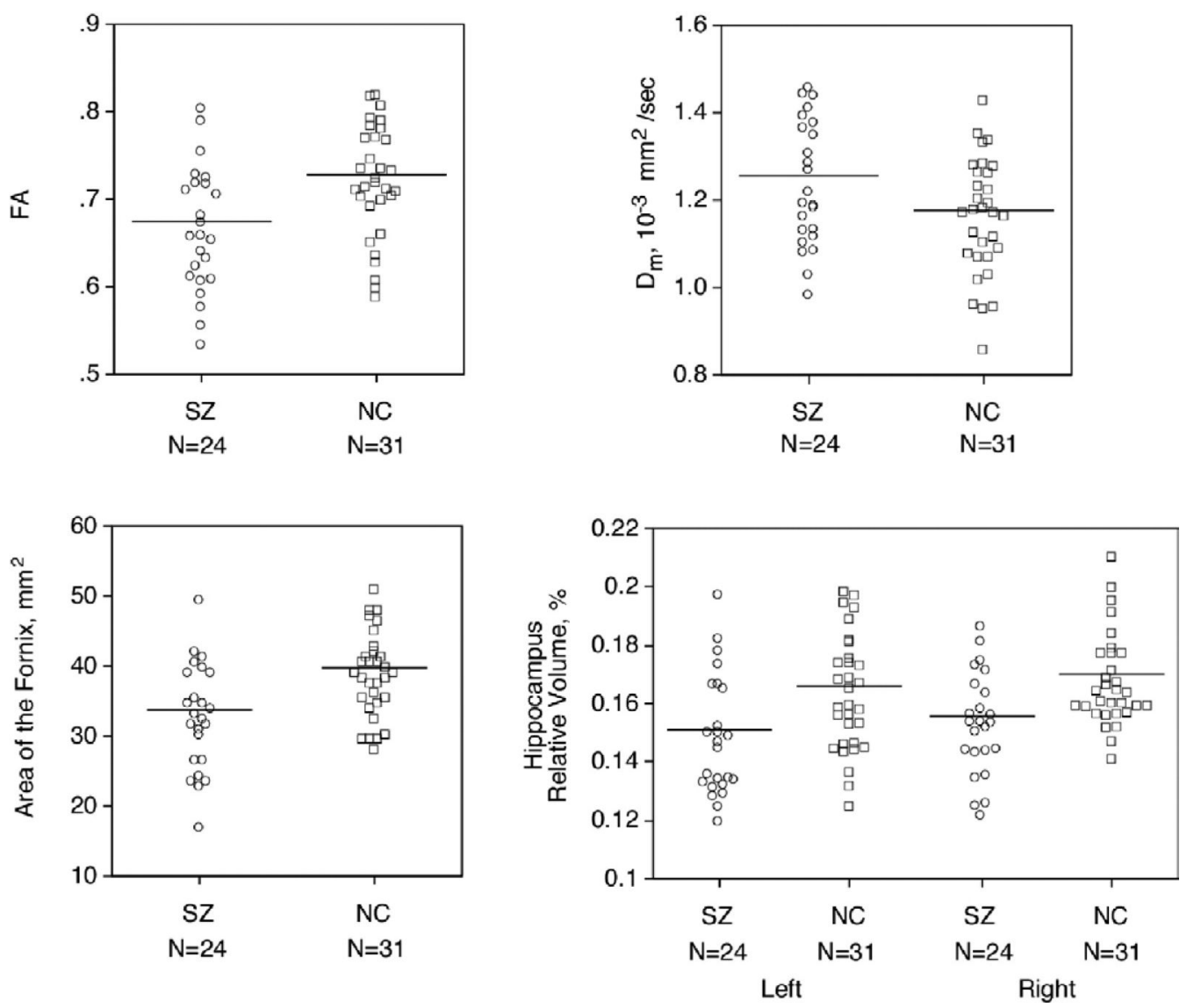

Figure 3.

Scattergrams of fractional anisotropy, $\mathrm{D}_{\mathrm{m}}$, cross-sectional area of the fornix, and relative volume of left and right hippocampus. Mean values are indicated by horizontal bars. SZ, schiophrenia group; NC, normal control group. 


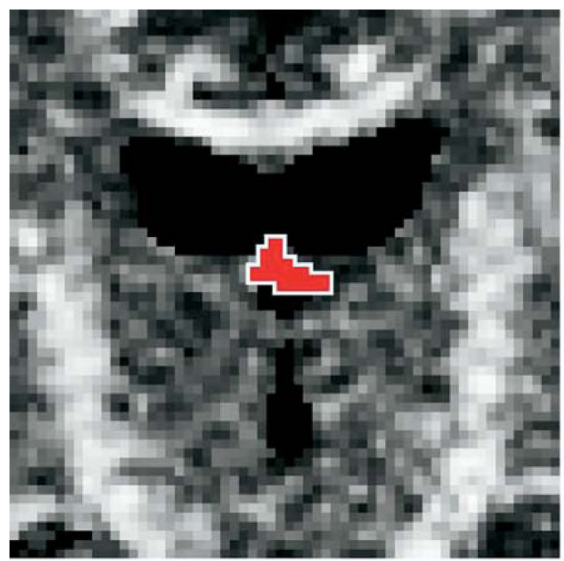

SZ

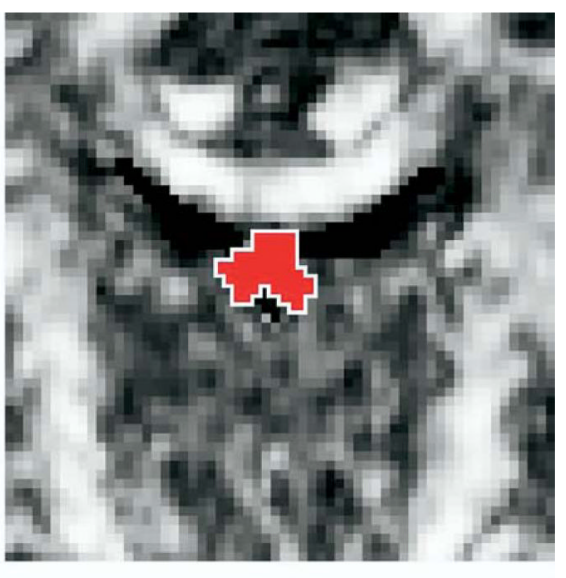

NC

Figure 4.

Fractional anisotropy map of a schizophrenic patient (SZ, left) and control subject (NC, right). Red color area with a white outline shows the delineated fornix. 

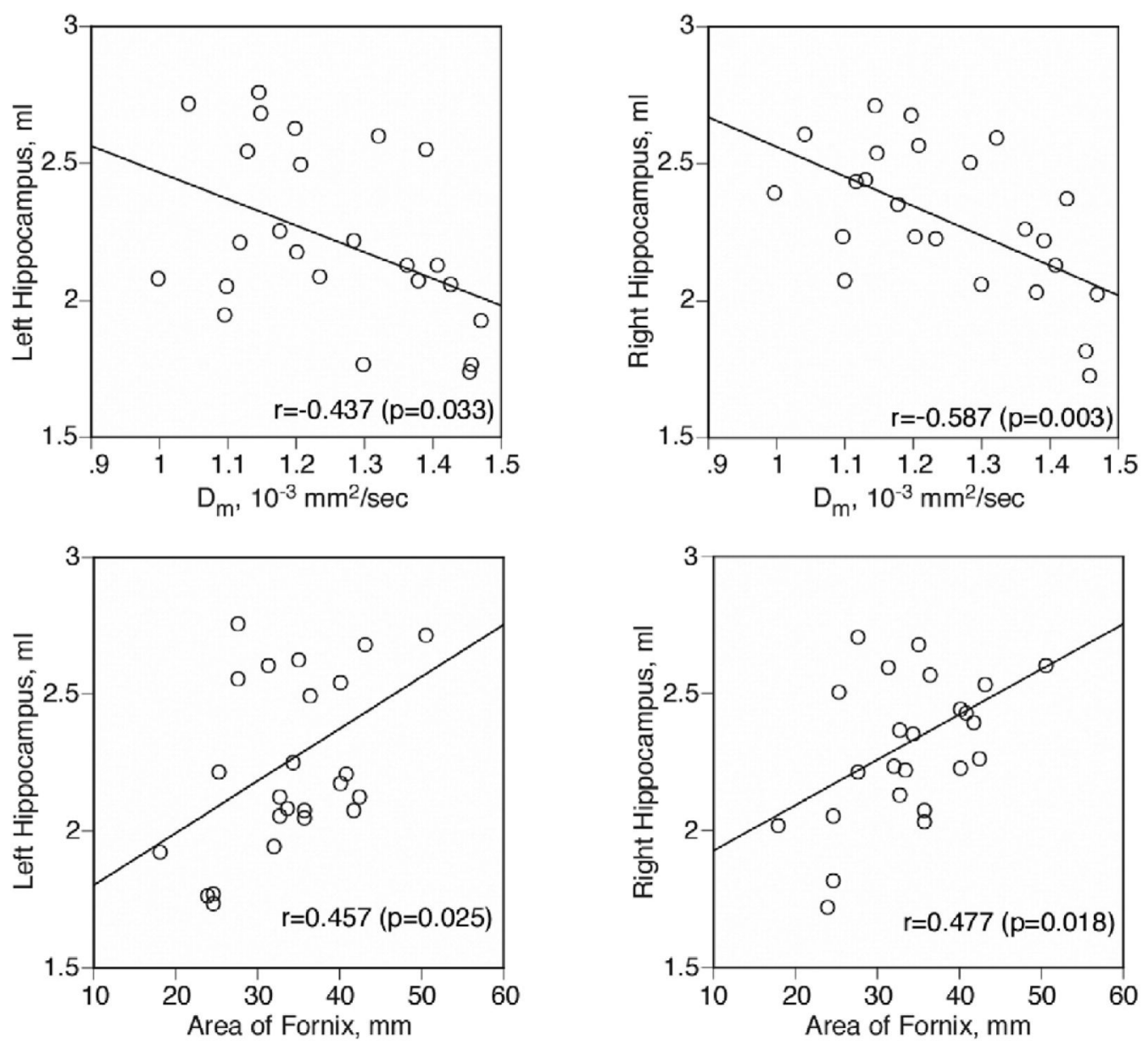

Figure 5.

Scattergrams between $\mathrm{D}_{\mathrm{m}}$ and absolute volume of left and right hippocampus between crosssectional area of the fornix in patients with schizophrenia. Pearson's Product-Moment correlation coefficients were used for testing statistical significance, and a least squares line is also shown. 

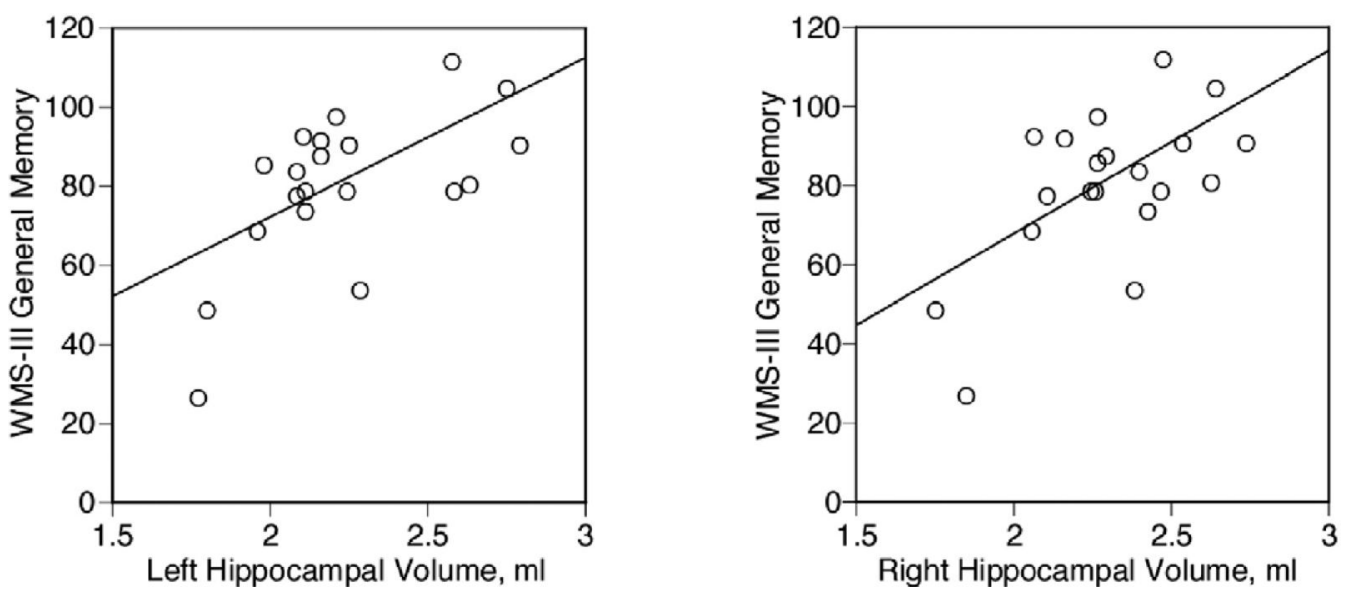

Figure 6.

Scattergrams between absolute volume of left and right hippocampus and general memory index scores of Wechsler Memory Scale-3rd edition (WMS-III) in patients with schizophrenia. Spearman's rho was used for testing statistical significance; we also plotted a least squares line for the convenience of the reader. 


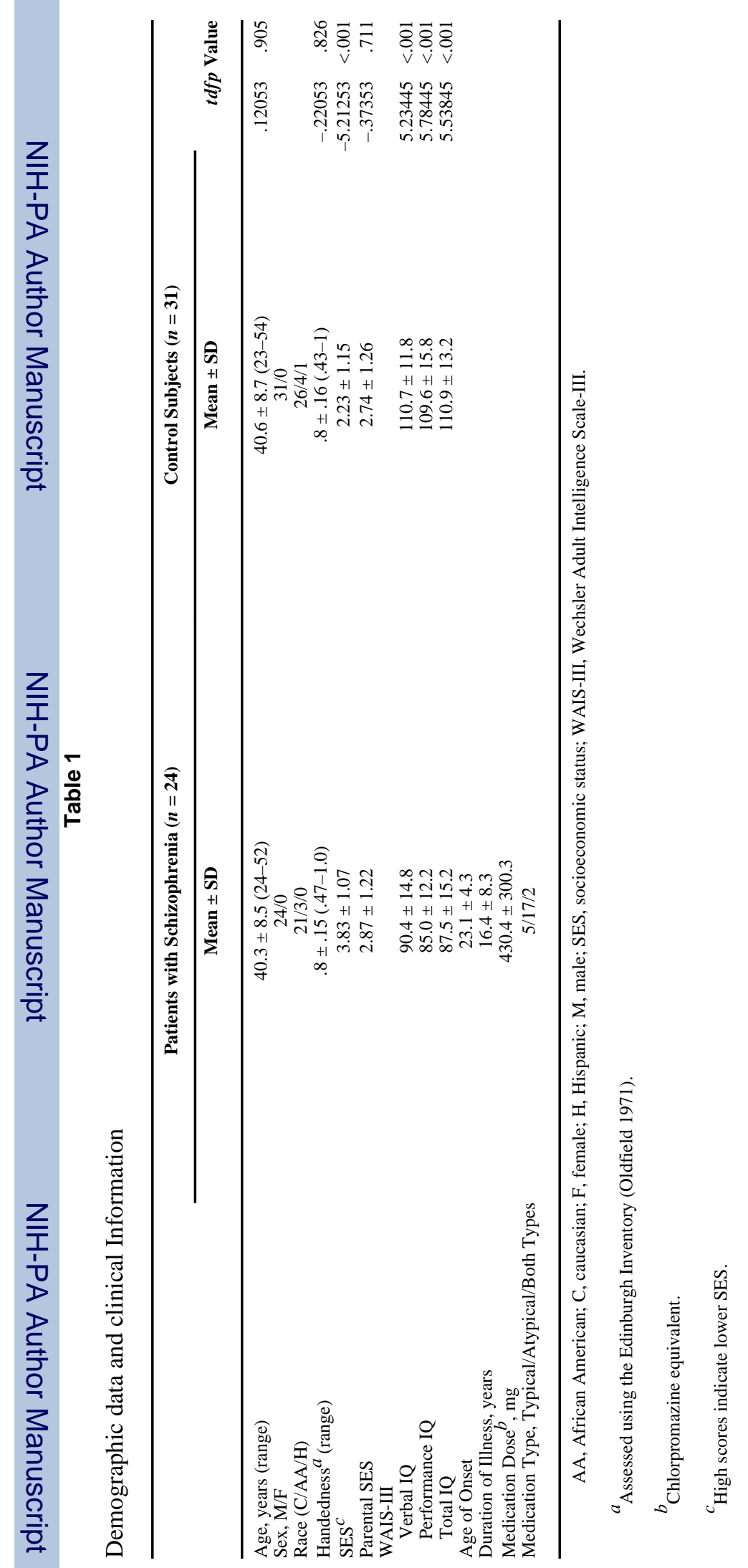

Biol Psychiatry. Author manuscript; available in PMC 2009 October 27. 


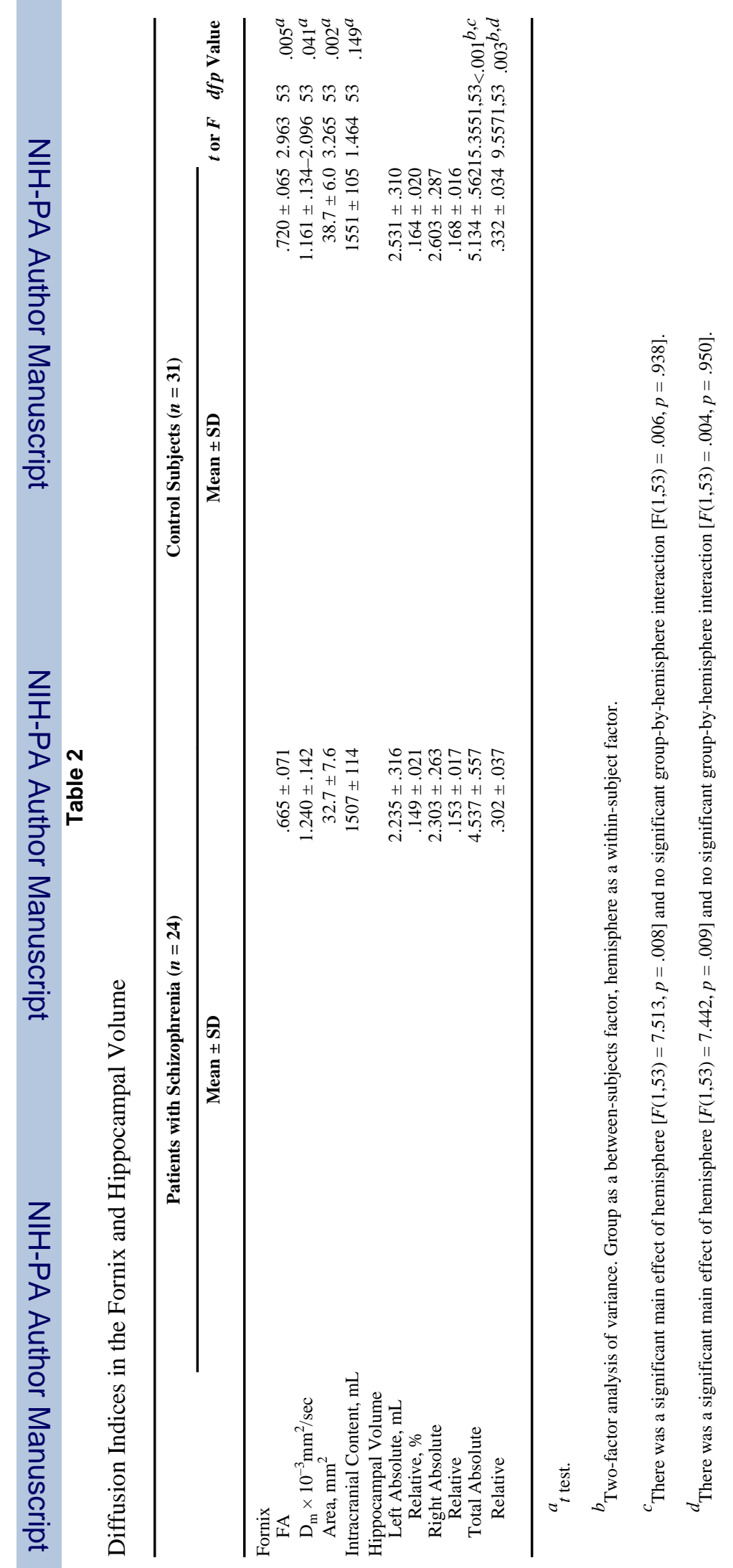

Biol Psychiatry. Author manuscript; available in PMC 2009 October 27. 